Prof. Dr. med.
$\begin{aligned} & \text { H. S. FüeßI } \\ & \text { Isar-Amper- } \\ & \text { Klinikum, } \\ & \text { Kl. München-Ost, } \\ & \text { Haar }\end{aligned}$

\title{
Die neue Sprache der Medizin und ihre Folgen
}

\begin{abstract}
Ganz allmählich und für viele unbemerkt hat sich in der Gesellschaft eine neue Sprache im Gesundheitswesen eingebürgert. Vielleicht charakterisiert diese Veränderung mehr als alle tiefschürfenden Analysen die Entwicklung der Medizin.
\end{abstract}

- Wir leben in einer ökonomischen Krise des Gesundheitswesens. Politik, Wirtschaft und Gesellschaft versuchen, den rasanten Kostenanstieg im System zu kontrollieren. Zeitgleich hat sich die Umgangssprache verändert: Aus Patienten (ehemals „Leidende“) sind Kunden oder Verbraucher (consumer), aus Ärzten bzw. „Doktoren“, die früher lehrten (docere), Versorger (provider) geworden. Der Patient ist der Käufer, der Arzt der Verkäufer oder Händler.

Natürlich ist Krankheit und ihre Behandlung mit finanziellen Aspekten verknüpft, und der Arzt muss für seine Tätigkeit bezahlt (früher honoriert) werden. Doch die neue Sprache reduziert die Arzt-Patienten-Beziehung auf den finanziellen Aspekt. Gleichzeitig gehen die über Jahrhunderte gültigen Aspekte ärztlicher Tätigkeit, nämlich die notwendige psychologische, geistige und humanistische Dimension verloren bzw. erübrigen sich. Hinzu kommt, dass der übergeordnete Begriff des "Versorgers"

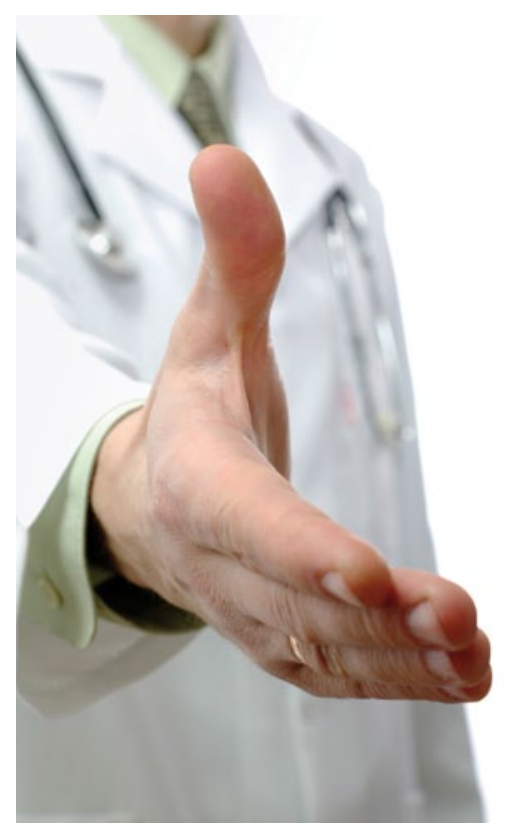

Verkommt der Arzt zum Verkäufer?

keinen Spielraum bereit hält für die spezielle Ausbildung, für Erfahrung und Fähigkeiten. Die neue Sprache degradiert Gesundheit zu einer vorgefertigten Ware aus dem Kaufhausregal, deren Verkauf keiner personellen, dynamischen oder fachlichen Expertise bedarf.

- P. Hartzband, J. Groopman

The new language in medicine. New Engl. J. Med. 365 (2011) 1372-1373

\section{Kommentar}

Über Jahrhunderte wurden Ärzte, die nach Gewinn strebten, verachtet, ja sogar verspottet, z. B. in den Komödien von Moliere. Die neue Sprache ist unter dem Druck der Kostenexplosion im Gesundheitswesen entstanden und offensichtlich dem Unternehmertum entlehnt. Sie impliziert eine völlig neue Sichtweise auf das Gesundheitswesen. Heute werden alle Beteiligten als erfolgreiche und angesehene Unternehmer charakterisiert, wenn sie die Technik der Gewinnmaximierung für das System, die Klinik und sich selbst beherrschen.

Wie werden sich das neue Vokabular und die zugrunde liegende Einstellung auf die zukünftige Generation der im Gesundheitswesen Tätigen auswirken? Das als unabdingbar bezeichnete, besondere Arzt-PatientenVerhältnis wird sich erübrigen, wenn die Umgangssprache den Arzt zum Kaufmann und die Medizin auf die ökonomischen Aspekte reduziert. Es steht auch zu befürchten, dass eine Medizin, die aus Sicht der Allgemeinheit auf industrielle und ökonomische Aspekte reduziert wird, weniger kreative und unabhängig denkende Menschen mit Interesse an Wissenschaft, Biologie, Humanität und Fürsorge anziehen wird.

H. HOLZGREVE = 